\section{There Is No Evidence to Support the Linear No-Threshold Model of Radiation Carcinogenesis}

TO THE EDITOR: Despite our previous responses $(1,2)$, Duncan et al. continue to offer assumptions and misconceptions, instead of evidence and rational argument. For example, they make the following 4 claims in their most recent Letter to the Editor (3):

1. They continue to assert that most DNA double-strand breaks caused by ionizing radiation are repaired by nonhomologous end joining, an error-prone process. Such cells survive but are left with mutations as permanent "information scars," and there is little, if any, evidence, they say, that these cells are later removed to eliminate the low but finite risk of future cancers. As we noted previously (1), the repair fidelity of the damage produced by low-dose, low-LET (linear energy transfer) radiation associated with medical imaging may be no less than that by homologous recombination for endogenously induced damage. This is because the damage produced endogenously and by low-dose, low-LET radiation (whether isolated double-strand breaks or other) occurs by the same mechanism-free radicals; the predominant mode of interaction of low-LET radiation is by indirect action.

2. Duncan et al. quote us correctly as saying that mutations are not sufficient to produce clinical cancer. They then counterpose a study by Martincorena and Campbell (4) that they say shows that cancers are necessarily preceded by mounting numbers of mutations in the same cell. These are both true. That multiple mutations are necessary does not say that they are sufficient to produce clinical cancer. Only epidemiologic studies, of which there are many, that demonstrate lowered cancer rates among those exposed to low-dose radiation can decide the issue, as logical as assumptions about outcome may appear.

3. Duncan et al. cite in vitro experiments to justify their claim that surviving cells after radiation exposure contain DNA mutations that are not "removed with sufficient reliability to eliminate the low but finite risk of future cancers." They refer to these in vitro experiments as "elegant," and although they may be elegant for limited purposes, in vitro data cannot be considered indicative of cancer development in intact organisms (5). In vitro experiments lack both the mitochondrial oxygen-metabolizing processes that produce continual and extensive DNA damage every second of every day and the immune systems that continually remove cells that could potentially initiate cancer development as well as the cells that have begun that process. If this were not true of immune surveillance, we would all develop cancer. Intact organisms possess a steady state of DNA-damaged cells that permit adaptive processes, stimulated further by low-dose radiation, to repair or remove not only most of the added DNA damaged cells due to the radiation, but also some of the preexisting steady-state DNA-damaged cells. The net effect is a decrease in the number of damaged cells relative to the preexposure steady-state number. That such repair or removal may not be $100 \%$ efficient is correct, but it is

COPYRIGHT @ $\odot 2018$ by the Society of Nuclear Medicine and Molecular Imaging. incomplete when mention of the steady-state preexposure damage level is omitted from the argument.

4. Duncan et al. claim that radiation-induced mutations accrue over time, irrespective of dose rate. To support this claim, a 2012 article is cited that according to them "nicely" summarizes and putatively provides "compelling" evidence that cancer risk increases with protracted, low-dose exposure based on several epidemiologic studies. However, the author of the cited article notes that these studies "do not put the debate over low-risk radiation to rest" and that the dose response in the range of $0-100 \mathrm{mSv}$ is still an open question. Further, the 3 studies described have more recently all been shown to contain fatal errors.

The search for the truth requires a critical reading of the literature, not cherry-picking studies that do not stand up to critical scrutiny and uncritically accepting proclamations by recognized voices of authority devoid of evidence. The main problem here is that there is a conceptual fork in the road where one has to choose which path to take.

One path follows adaptive protection studies into an ever-expanding world in which the generated questions permit experiments and discoveries with respect to new uses of below-threshold exposures and the mechanisms whereby such stimulated protections occur. The other path is a static nonquestion-generating world in which regulations proliferate and govern ways of protecting the public and radiation workers against imaginary harms, of diverting untold funds from real public health needs, and robbing us of proven health-enhancing procedures. And instead of stimulating regulatory policies that protect the public against actual potential harms, they stimulate unwarranted and widespread fear of radiation among both the public and the medical personnel, including diagnostic imaging professionals.

Readers, which path do you choose?

\section{REFERENCES}

1. Siegel JA, Sacks B, Pennington CW, Welsh JS. DNA repair following exposure to ionizing radiation is not error-free: but this does not increase cancer incidence or mortality. J Nucl Med. 2018;59:349.

2. Siegel JA, Sacks B, Greenspan B. Radiation dose does indeed matter: proof that invalidates the linear no-threshold model. J Nucl Med. 2018;59:1779-1780.

3. Duncan JR, Lieber MR, Adachi N, Wahl R. Radiation dose does matter: mechanistic insights into DNA damage and repair support the linear no-threshold model of lowdose radiation health risks [reply]. J Nucl Med. 2018;59:1780-1781.

4. Martincorena I, Campbell PJ. Somatic mutation in cancer and normal cells. Science. 2015;349:1483-1489.

5. Siegel JA, Greenspan BS, Maurer AH, et al. The BEIR VII estimates of low-dose radiation health risks are based on faulty assumptions and data analyses: a call for reassessment. J Nucl Med. 2018;59:1017-1019.

\author{
Jeffry A. Siegel* \\ Bill Sacks \\ Bennett S. Greenspan \\ *Nuclear Physics Enterprises \\ 4 Wedgewood Dr. \\ Marlton, NJ 08053 \\ E-mail:nukephysics@comcast.net
}

Published online Oct. 25, 2018.

DOI: 10.2967/jnumed.118.221515 